\title{
Revision ambulante Tarifstruktur: Tarifpartnerschaft statt Amtstarif
}

\section{Marina Lüscher}

FMH, Abteilung Ambulante Tarife und Verträge Schweiz

Im Mittelpunkt der Informationsveranstaltung vom 4. November 2015 standen Informationen zur Revision der ambulanten Tarifstruktur für medizinische Leistungen. Kernbotschaft dieser Tagung: Wahrung der Tarifautonomie und die Einführung des revidierten Tarifs im Jahr 2017.

\section{Revision der ambulanten Tarifstruktur: Warum?}

Referent: Dr. med. J. Schlup, Präsident FMH Weshalb braucht es eine Revision des derzeitigen ambulanten Tarifs? Mit dieser Frage eröffnete Jürg Schlup sein Referat. Die Eidgenössische Finanzkontrolle (EFK) hat 2008 mit der Evaluation des TARMED begonnen. In ihrem Bericht von 2010 wurde evaluiert, ob die mit der Einführung der Tarifstruktur TARMED gesetzten Ziele erreicht wurden und inwieweit Optimierungspotential bestehe. Der EFK-Bericht enthält einige zentrale Kritikpunkte, wie zum Beispiel die fehlende Differenzierung von Zeit- und Handlungsleistungen, nicht plausibilisierte Minutagen, nicht nachvollziehbare Pro-

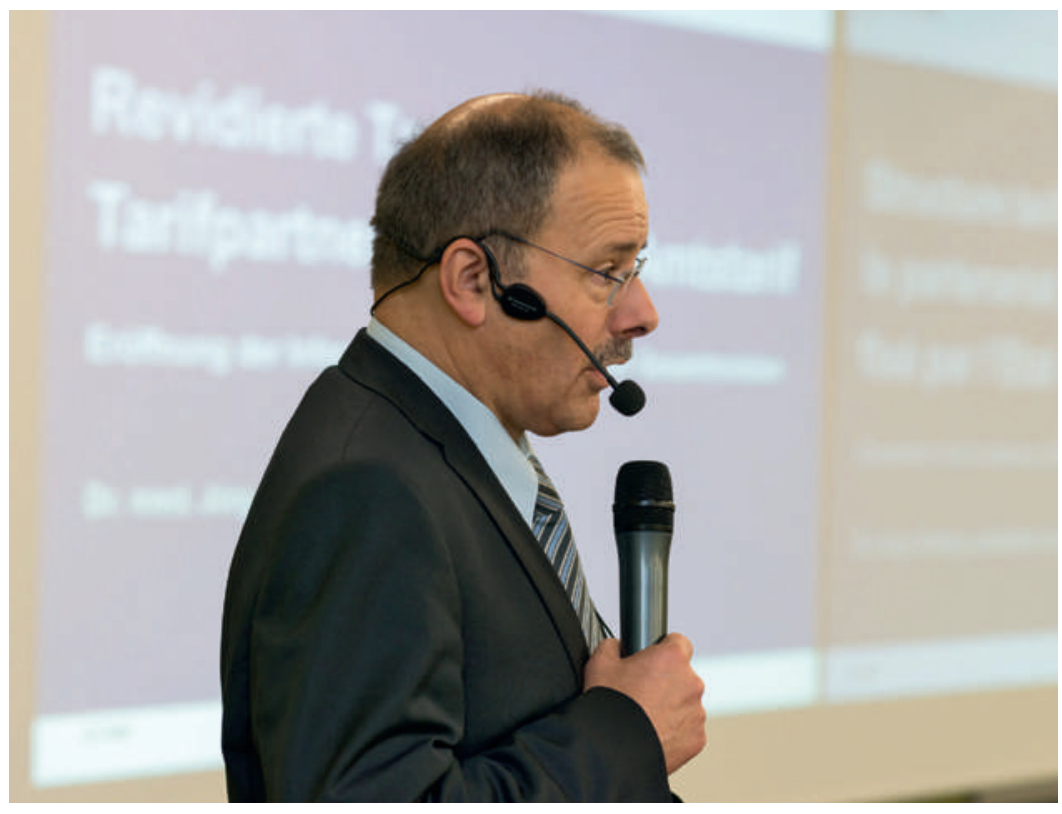

Dr. med. Jürg Schlup, Präsident der FMH, eröffnet die Informationsveranstaltung. duktivitätsfaktoren und die Verrechnung von Analogiepositionen. Ausserdem sei die Basis für die Taxpunkte zum Teil veraltet und die Grundversorger werden benachteiligt.

Die EFK empfiehlt in ihrem Bericht dem Bundesrat, seine gesetzliche Kompetenz besser zu nutzen und bei fehlender Einigung der Tarifpartner eine vorläufige Tarifierungslösung durchzusetzen. Diese Empfehlung war auch die Grundlage für die Verordnungsanpassung des KVG-Artikels 43 Ziffer 5bis, in dem der Bundesrat explizit berechtigt wird, einen Tarifeingriff vorzunehmen, falls diese nicht mehr sachgerecht ist. J. Schlup betont in seinem Fazit, dass weitere Eingriffe folgen werden, falls es uns nicht gelingt, eine gemeinsame, konsensfähige revidierte Tarifstruktur einzureichen.

\section{Revidierter Arzttarif: Stand der Arbeiten}

\section{Referent: Christian Oeschger, Projektleiter} Gesamtrevision ambulante Tarifstruktur, FMH Die Interessen und Vorstellungen aller Anspruchspersonen zu berücksichtigen ist die grösste Herausforderung in der Revision des ambulanten Tarifs. Mit der tripartiten Zusammenarbeit seit 2012 (FMH, H+ und MTK) wurde erreicht, was vor Jahren noch undenkbar war. Die meisten Fachteams stehen kurz vor dem Abschluss bzw. sind bereits abgeschlossen. Die Arbeitsgruppe Spartenkalkulation hat in einem ersten Schritt alle Sparten im Spitalmodell (INFRA-Modell) gerechnet. In einem zweiten Schritt werden die Sparten der ambulanten Praxis (KOREG-Modell) gerechnet und plausibilisiert.

Die Aktualisierung der Geräte sowie die Dotation des nichtärztlichen Personals in den Sparten werden in den entsprechenden Fachteams vorgestellt. 


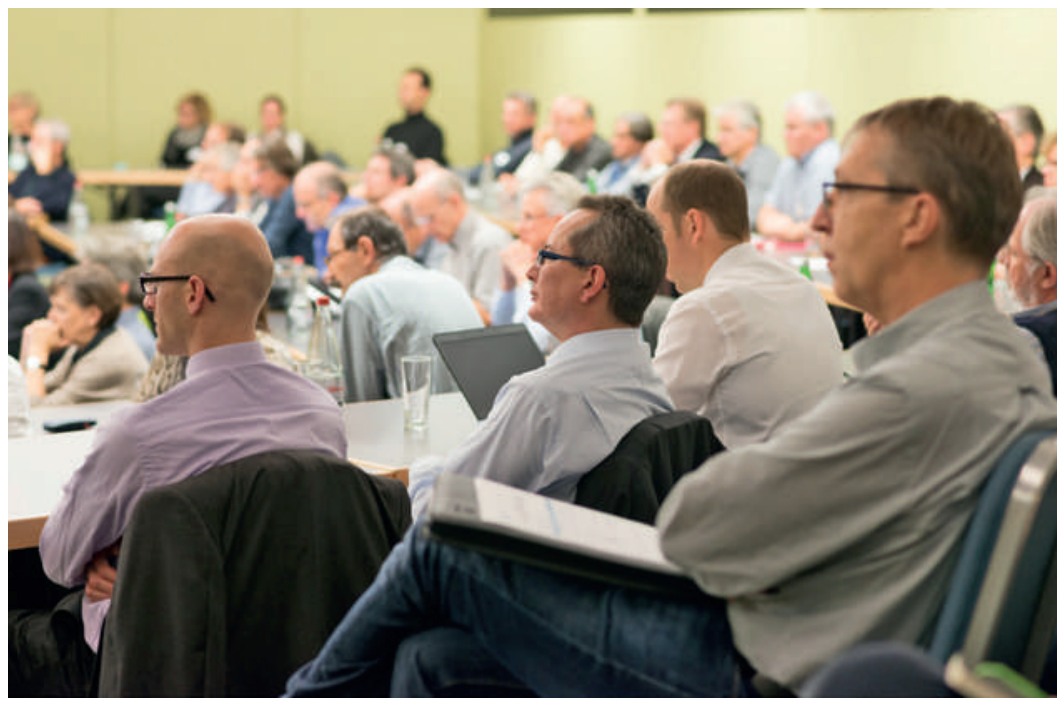

Das Interesse, an der Informationsveranstaltung der ambulanten Tarifstruktur teilzunehmen, war gross.

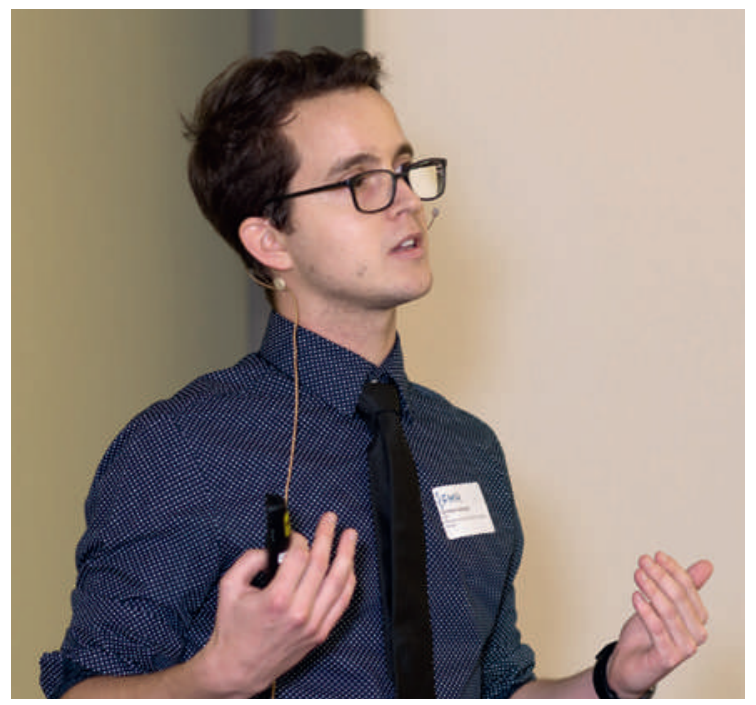

Christian Oeschger informiert über den aktuellen Stand der Arbeiten.

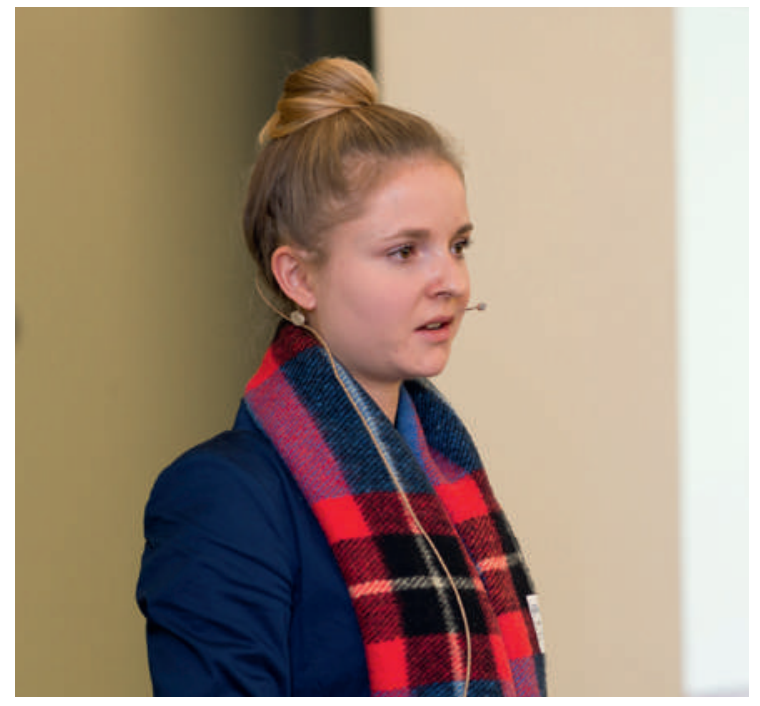

Den genauen Ablauf des Vernehmlassungsprozesses gibt Livia Walker bekannt.

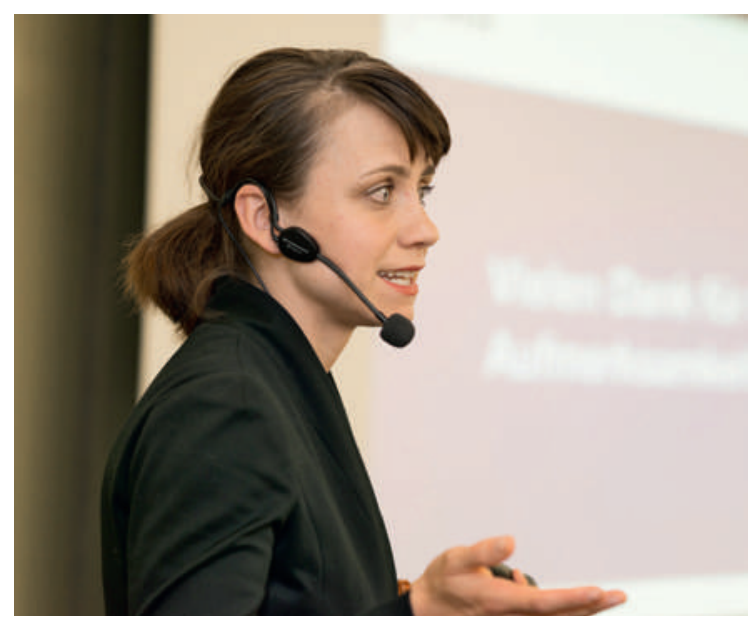

Kerstin Schutz berichtet über den Vernehmlassungsprozess im Allgemeinen. 


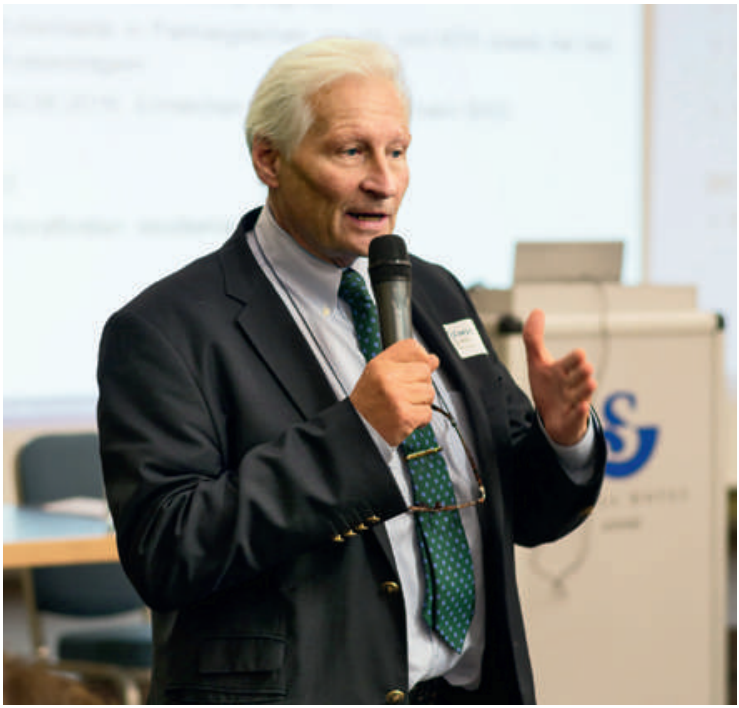

Dr. med. Urs Stoffel führt durch die zweite Hälfte der Informationsveranstaltung.

\section{Quantitative Dignitäten}

\section{Referent: Dr. med. Urs Stoffel, Zentralvorstand} «Schaffung eines Gefässes zur Abbildung eines Wertesystems, das als Grundlage einer Preismodulation der ärztlichen Leistung dienen soll.»

Die Dignitäten gehören in der Revision zu den heissesten Eisen und sind ein umstrittenes Thema. Einer der Hauptkritikpunkte am heutigen TARMED sind die Ungleichbehandlung der verschiedenen medizinischen Disziplinen sowie fehlende Entwicklungsmöglichkeiten des ärztlichen Einkommens in der Praxis.

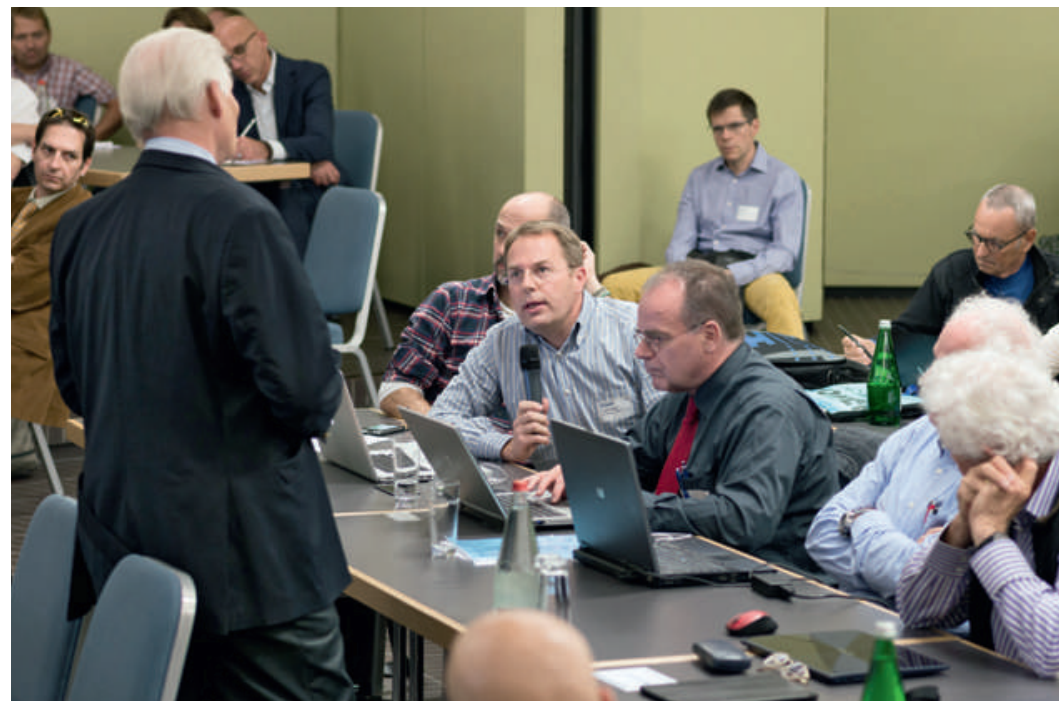

Es finden angeregte Diskussionen zwischen den Referenten und den Teilnehmern statt.
Auf Ebene der Dachorganisationen der FMH konnte man sich diesbezüglich bisher auf kein neues Konzept einigen, weshalb die FMH in den vergangenen Wochen einen eigenen Vorschlag ausgearbeitet hat.

Das aktuelle Konzept der quantitativen Dignitäten ist überholt und nicht mehr sachgerecht. Ursprünglich wurde über die quantitative Dignität der unterschiedlichen Ausbildungsdauer und damit den sogenannten «Hungerjahren» in der medizinischen Ausbildung Rechnung getragen. Das heisst, dass ärztliche Leistungen im TARMED unterschiedlich bewertet wurden. Im Ergebnis mussten die unterschiedlichen Bewertungen neutral sein, das heisst es gibt im TARMED Tarifpositionen, deren Ärztliche Leistung (AL) mit einem Faktor über 1, und andere, die mit einem Faktor unter 1 multipliziert werden.

Das technische Gedankenmodell zum Ersatz der quantitativen Dignität in der Tarifstruktur und weitere Forderungen, die häufig mit der Dignität in Verbindung gebracht werden, sehen stark vereinfacht Folgendes vor:

- An Stelle der quantitativen Dignitäten tritt ein individueller Faktor für den Arzt, der verschiedenste Argumente, wie beispielsweise Praxisstandort, Qualitätssicherung, Berufserfahrung, aber auch klassische Argumente wie die Weiterbildungszeit beinhalten würde.

- Die bisherigen quantitativen Dignitäten in der Tarifstruktur werden auf 1 gesetzt und damit neutralisiert.

- Für die Erarbeitung eines solchen, breit abgestützten Wertesystems ausserhalb der eigentlichen Tarifstruktur rechnet Dr. Stoffel mit rund einem Jahr Entwicklungszeit. Die Eckwerte, Argumente und die Gewichtung der Argumente müssen durch die Ärzteschaft gemeinsam erarbeitet werden und können nicht einfach als Gesamtmodell eingekauft werden.

Bevor die Teilnehmenden die Informationsveranstaltung bei einem Apéro ausklingen liessen, fasste U. Stoffel in einem Rückblick die wichtigsten Statements zusammen und unterstrich noch einmal die relevanten Punkte im Zusammenhang mit der Revision der ambulanten medizinischen Leistungen. Er appellierte an die Anwesenden, dass wir es nur gemeinsam schaffen, und dazu braucht es die Kompromissbereitschaft aller.

Alle Referate und Publikationen finden Sie auf unserer Webseite www.fmh.ch $\rightarrow$ myfmh $\rightarrow$ Publikationen $\rightarrow$ Tarifdelegierten-Tag. 